\title{
Some Results on Prime and $k$-Prime Labeling
}

\author{
S. K. Vaidya (Corresponding author) \\ Department of Mathematics, Saurashtra University \\ Rajkot 360005, India \\ E-mail: samirkvaidya@yahoo.co.in \\ U. M. Prajapati \\ Department of Mathematics, St. Xavier's College \\ Ahmedabad 380009, India \\ E-mail: udayan64@yahoo.com
}

\begin{abstract}
A graph $G=(V, E)$ with $n$ vertices is said to admit prime labeling if its vertices can be labeled with distinct positive integers not exceeding $n$ such that the labels of each pair of adjacent vertices are relatively prime. A graph $G$ which admits prime labeling is called a prime graph. In the present work we investigate some classes of graphs which admit prime labeling. We also introduce the concept of $k$-prime labeling and investigate some results concern to it. This work is a nice combination of graph theory and elementary number theory.
\end{abstract}

Keywords: Graph Labeling, Prime labeling, Prime graph, $k$-prime labeling, $k$-prime graph

\section{Introduction}

We begin with simple, finite, undirected and non-trivial graph $G=(V, E)$ with the vertex set $V$ and the edge set $E$. The number of elements of $V$, denoted as $|V|$ is called the order of the graph $G$ while the number of elements of $E$, denoted as $|E|$ is called the size of the graph $G$. In the present work $C_{n}$ denotes the cycle with $n$ vertices and $P_{n}$ denotes the path of $n$ vertices. In the wheel $W_{n}=C_{n}+K_{1}$ the vertex corresponding to $K_{1}$ is called the apex vertex and the vertices corresponding to $C_{n}$ are called the rim vertices. The graph $f_{n}=P_{n-1}+K_{1}$ is called a fan and the vertex corresponding to $K_{1}$ is called the apex vertex of the fan. For various graph theoretic notations and terminology we follow (Gross, J. \& Yellen, J., 2004) whereas for number theory we follow (Burton, D. M.,1990). We will give brief summary of definitions and other information which are useful for the present investigations.

Definition 1.1 If the vertices of the graph are assigned values subject to certain conditions then it is known as graph labeling.

For latest survey on graph labeling we refer to (Gallian, J. A., 2009). Vast amount of literature is available on different types of graph labeling and more than 1000 research papers have been published so far in last four decades. For any graph labeling problem following three features are really noteworthy:

- a set of numbers from which vertex labels are chosen;

- a rule that assigns a value to each edge;

- a condition that these values must satisfy.

The present work is aimed to discuss one such labeling known as prime labeling.

Definition 1.2 A prime labeling of a graph $G$ is an injective function $f: V \rightarrow\{1,2, \cdots,|V|\}$ such that for every pair of adjacent vertices $u$ and $v, \operatorname{gcd}(f(u), f(v))=1$. The graph which admits prime labeling is called a prime graph.

The notion of prime labeling was originated by Entringer and was discussed in (Tout, A., 1982, p. 365-368). Many researchers have studied prime graphs. It has been proved by $\left(\mathrm{Fu}, \mathrm{H}\right.$. L., 1994, p. 181-186) $P_{n}$ on $n$ vertices is a prime graph. It has been proved by (Lee, S. M., 1988, p. 59-67) wheel graph $W_{n}$ is a prime graph if and only if $n$ is even. In (Deretsky, T. D., 1991, p. 359-369) cycle $C_{n}$ is a prime graph.

Definition 1.3 The graph $G=\left\langle W_{n}: W_{m}\right\rangle$ is the graph obtained by joining apex vertices of wheels $W_{n}$ and $W_{m}$ to a new vertex $w$.

Definition 1.4 A t-ply $P_{t}(u, v)$ is a graph with $t$ paths, each of length at least two and such that no two paths have a vertex in common except for the end vertices $u$ and $v$.

Definition 1.5 Two prime integers are said to be twin primes if they differ by 2 . 


\section{Some Results on Prime Graphs}

Theorem 2.1 Let $n_{1}$ and $n_{2}$ be two even positive integers such that $n_{1}+n_{2}+3=p$ where $p$ and $p-2$ are twin primes. Then the graph $G=<W_{n_{1}}: W_{n_{2}}>$ is a prime graph.

Proof: Let $u_{1}, u_{2}, \cdots, u_{n_{1}}$ be the consecutive rim vertices of $W_{n_{1}}$ and $v_{1}, v_{2}, \cdots, v_{n_{2}}$ be the consecutive rim vertices of $W_{n_{2}}$. Let $u_{0}$ and $v_{0}$ be the apex vertices of $W_{n_{1}}$ and $W_{n_{2}}$ respectively which are adjacent to a new common vertex $w$.

Define $f: V \longrightarrow\{1,2,3, \cdots,|V|\}$ as follows:

$$
\begin{aligned}
& f\left(u_{i}\right)=1+i, \forall i=0,1,2, \cdots, n_{1} ; \\
& f\left(v_{0}\right)=p ; \\
& f\left(v_{j}\right)=1+n_{1}+j, \forall j=1,2,3, \cdots, n_{2} \text { and } \\
& f(w)=p-1 .
\end{aligned}
$$

Then clearly $f$ is an injection.

For an arbitrary edge $e=a b$ of $G$ we claim that $\operatorname{gcd}(f(a), f(b))=1$.

To prove our claim the following cases are to be considered.

1. If $e$ is an edge of $W_{n_{1}}$ then we have the following possibilities:

- if $e=u_{j} u_{j+1}$ for some $j \in\left\{1,2, \cdots, n_{1}-1\right\}$ then $\operatorname{gcd}\left(f\left(u_{j}\right), f\left(u_{j+1}\right)\right)=\operatorname{gcd}(1+j, 1+j+1)=\operatorname{gcd}(j+1, j+2)=1$ as $j+1$ and $j+2$ are consecutive positive integers;

- if $e=u_{n_{1}} u_{1}$ then $\operatorname{gcd}\left(f\left(u_{n_{1}}\right), f\left(u_{1}\right)\right)=\operatorname{gcd}\left(n_{1}+1,2\right)=1$ as $n_{1}+1$ is an odd integer;

- if $e=u_{0} u_{j}$ for some $j \in\left\{1,2, \cdots, n_{1}\right\}$ then $\operatorname{gcd}\left(f\left(u_{0}\right), f\left(u_{j}\right)\right)=\operatorname{gcd}(1, j+1)=1$.

2. If $e=u_{0} w$ then $\operatorname{gcd}\left(f\left(u_{0}\right), f(w)\right)=\operatorname{gcd}(1, p-1)=1$.

3. If $e=v_{0} w$ then $\operatorname{gcd}\left(f\left(v_{0}\right), f(w)\right)=\operatorname{gcd}(p, p-1)=1$ as $p$ and $p-1$ are consecutive positive integers.

4. If $e$ is an edge of $W_{n_{2}}$ then we have the following possibilities:

- if $e=v_{j} v_{j+1}$ for some $j \in\left\{1,2,3, \cdots, n_{2}-1\right\}$ then $\operatorname{gcd}\left(f\left(v_{j}\right), f\left(v_{j+1}\right)\right)=\operatorname{gcd}\left(1+n_{1}+j, 1+n_{1}+j+1\right)=1$ as $1+n_{1}+j$ and $1+n_{1}+j+1$ are consecutive positive integers;

- if $e=v_{n_{2}} v_{1}$ then $\operatorname{gcd}\left(f\left(v_{n_{2}}\right), f\left(v_{1}\right)\right)=\operatorname{gcd}\left(1+n_{1}+n_{2}, n_{1}+2\right)=$ $\operatorname{gcd}\left(p-2, n_{1}+2\right)=1$ as $p-2$ is a prime number greater than $n_{1}+2$;

- if $e=v_{0} v_{j}$ for some $j \in\left\{1,2, \cdots, n_{2}\right\}$ then $\operatorname{gcd}\left(f\left(v_{0}\right), f\left(v_{j}\right)\right)=$ $\operatorname{gcd}\left(p, 1+n_{1}+j\right)=1$ as $p$ is a prime number greater than $1+n_{1}+j$.

Thus in each of the possibilities the graph $G$ under consideration admits a prime labeling. i.e. $G$ is a prime graph.

Illustration 2.2 A prime labeling of $\left\langle W_{10}: W_{6}>\right.$ is shown in Figure 1.

Theorem 2.3 If $n_{1} \geq 4$ is an even integer and $n_{2} \in N$ then the disjoint union of the wheel $W_{n_{1}}$ and the path graph $P_{n_{2}}$ is a prime graph.

Proof: Let $u_{0}$ be the apex vertex, $u_{1}, u_{2}, \cdots, u_{n_{1}}$ be the consecutive rim vertices of $W_{n_{1}}$ and $v_{1}, v_{2}, \cdots, v_{n_{2}}$ be the consecutive vertices of $P_{n_{2}}$. Let $G$ be the disjoint union of $W_{n_{1}}$ and $P_{n_{2}}$.

Define $f: V \rightarrow\{1,2,3, \cdots,|V|\}$ as follows:

$f\left(u_{i}\right)=1+i, \forall i=0,1,2, \cdots, n_{1} ;$ and $f\left(v_{j}\right)=n_{1}+1+j, \forall j=1,2,3, \cdots, n_{2}$.

Then obviously $f$ is an injection.

For an arbitrary edge $e=a b$ of $G$ we claim that $\operatorname{gcd}(f(a), f(b))=1$ due to following reasons:

1. If $e$ is an edge of $W_{n_{1}}$ such that $e=u_{0} u_{i}$ for some $i \in\left\{1,2,3, \cdots, n_{1}\right\}$ then $\operatorname{gcd}\left(f\left(u_{0}\right), f\left(u_{i}\right)\right)=\operatorname{gcd}\left(1, f\left(u_{i}\right)\right)=1$;

2. If $e$ is an edge of $W_{n_{1}}$ such that $e=u_{i} u_{i+1}$ for some $i \in\left\{1,2,3, \cdots, n_{1}-1\right\}$ then $\operatorname{gcd}\left(f\left(u_{i}\right), f\left(u_{i+1}\right)\right)=\operatorname{gcd}(1+i, 1+$ $i+1)=1$ as every pair of consecutive positive integers are relatively prime; 
3. If $e$ is an edge of $W_{n_{1}}$ such that $e=u_{n_{1}} u_{1}$ then $\operatorname{gcd}\left(f\left(u_{n_{1}}\right), f\left(u_{1}\right)\right)=\operatorname{gcd}\left(n_{1}+1,2\right)=1$ because $n_{1}+1$ is an integer;

4. If $e$ is an edge of $P_{n_{2}}$ such that $e=v_{j} v_{j+1}$ for some $j \in\left\{1,2, \cdots, n_{2}-1\right\}$. Then $\operatorname{gcd}\left(f\left(v_{j}\right), f\left(v_{j+1}\right)\right)=\operatorname{gcd}\left(n_{1}+1+\right.$ $\left.j, n_{1}+1+j+1\right)=1$ as $n_{1}+1+j$ and $n_{1}+1+j+1$ are consecutive positive integers.

Thus in each of the possibilities the graph $G$ under consideration admits a prime labeling. i.e. $G$ is a prime graph. Illustration 2.4 A prime labeling of the disjoint union of $W_{8}$ and $P_{6}$ is shown in the Figure 2.

Theorem 2.5 If $n_{1} \geq 4$ is an even integer and $n_{2} \in N$ then the graph obtained by identifying any of the rim vertices of a wheel $W_{n_{1}}$ with an end vertex of a path graph $P_{n_{2}}$ is a prime graph.

Proof: Denote the apex vertex of $W_{n_{1}}$ by $u_{0}$ and the consecutive rim vertices of $W_{n_{1}}$ by $u_{1}, u_{2}, \cdots, u_{n_{1}}$. Let $v_{1}, v_{2}, \cdots, v_{n_{2}}$ be the consecutive vertices of $P_{n_{2}}$. Without loss of generality assume that the end vertex $v_{1}$ of $P_{n_{2}}$ is identified with the rim vertex $u_{n_{1}}$ of $W_{n_{1}}$. Define $f: V \rightarrow\{1,2,3, \cdots,|V|\}$ as follows: $f\left(u_{i}\right)=1+i, \forall i=0,1,2, \cdots, n_{1} ;$ and $f\left(v_{j}\right)=n_{1}+j$, $\forall j=2,3,4, \cdots, n_{2}$. Then obviously $f$ is an injection. For an arbitrary edge $e=a b$ of $G$ we claim that $\operatorname{gcd}(f(a), f(b))=1$ due to following reasons:

1. If $e$ is an edge of $W_{n_{1}}$ such that $e=u_{0} u_{i}$ for some $i \in\left\{1,2,3, \cdots, n_{1}\right\}$ then $\operatorname{gcd}\left(f\left(u_{0}\right), f\left(u_{i}\right)\right)=\operatorname{gcd}\left(1, f\left(u_{i}\right)\right)=1$;

2. If $e$ is an edge of $W_{n_{1}}$ such that $e=u_{i} u_{i+1}$ for some $i \in\left\{1,2,3, \cdots, n_{1}-1\right\}$ then for $i \neq n_{1}-1, \operatorname{gcd}\left(f\left(u_{i}\right), f\left(u_{i+1}\right)\right)=$ $\operatorname{gcd}(i+1, i+2)=1$ as $i+1$ and $i+2$ are consecutive positive integers;

3. If $e$ is an edge of $W_{n_{1}}$ such that $e=u_{n_{1}} u_{1}$ then $\operatorname{gcd}\left(f\left(u_{n_{1}}\right), f\left(u_{1}\right)\right)=\operatorname{gcd}\left(n_{1}+1,2\right)=1$ as $n_{1}+1$ is an odd positive integer;

4. If $e$ is an edge of $P_{n_{2}}$ such that $e=v_{j} v_{j+1}$ for some $j \in\left\{1,2, \cdots, n_{2}-1\right\}$. In this $\operatorname{case} \operatorname{gcd}\left(f\left(v_{j}\right), f\left(v_{j+1}\right)\right)=$ $\operatorname{gcd}\left(n_{1}+j, n_{1}+j+1\right)=1$ as $n_{1}+j$ and $n_{1}+j+1$ are consecutive positive integers.

Thus in each of the possibilities the graph $G$ under consideration admits a prime labeling. Which implies that $G$ is a prime graph.

Illustration 2.6 A prime labeling of the graph obtained by identifying an end vertex of $P_{6}$ with a rim vertex of $W_{8}$ is shown in the following Figure 3.

Theorem 2.7 If $n_{1}$ is even then the graph $G$ obtained by identifying the apex vertex of a wheel graph $W_{n_{1}}$ with an end vertex of $P_{n_{2}}$ is a prime graph.

Proof: Let $u_{0}$ be the apex vertex, $u_{1}, u_{2}, \cdots, u_{n_{1}}$ be the consecutive rim vertices of $W_{n_{1}}$ and $v_{1}, v_{2}, \cdots, v_{n_{2}}$ be the consecutive vertices of $P_{n_{2}}$. Without loss of generality assume that the end vertex $v_{1}$ of $P_{n_{2}}$ is identified with the apex vertex $u_{0}$ of $W_{n_{1}}$. Define $f: V \rightarrow\{1,2,3, \cdots,|V|\}$ as follows: $f\left(u_{i}\right)=1+i, \forall i=0,1,2, \cdots, n_{1}$; and $f\left(v_{j}\right)=n_{1}+j, \forall j=2,3,4, \cdots, n_{2}$. For an arbitrary edge $e=a b$ of $G$ we claim that $\operatorname{gcd}(f(a), f(b))=1$. Following reasons prove the claim.

1. if $e$ is an edge of $W_{n_{1}}$ then the restriction of $f$ on $\left\{1,2, \cdots,\left|V\left(W_{n_{1}}\right)\right|\right\}$ admits a prime labeling of $W_{n_{1}}$ as reported in (Lee, S. M., 1988). Thus $\operatorname{gcd}(f(a), f(b))=1$ for this edge $e$.

2. if $e$ is an edge of $P_{n_{2}}$ such that $e=v_{j} v_{j+1}$ for some $j \in\left\{1,2, \cdots, n_{2}\right\}$. In this case:

- for $j \neq 1, \operatorname{gcd}\left(f\left(v_{j}\right), f\left(v_{j+1}\right)\right)=\operatorname{gcd}\left(n_{1}+j, n_{1}+j+1\right)=1$ as $n_{1}+j$ and $n_{1}+j+1$ are consecutive positive integers.

- for $j=1, \operatorname{gcd}\left(f\left(v_{j}\right), f\left(v_{j+1}\right)\right)=\operatorname{gcd}\left(f\left(v_{1}\right), f\left(v_{2}\right)\right)=\operatorname{gcd}\left(1, n_{1}+2\right)=1$.

Thus in each of the possibilities the graph $G$ under consideration admits a prime labeling. Which implies that $G$ is a prime graph.

Illustration 2.8 A prime labeling of the graph defined by identifying an end vertex of $P_{6}$ with the apex vertex of $W_{8}$ is shown in the Figure 4.

Theorem 2.9 Let $G_{1}$ be a prime graph of order $n_{1}$ with a prime labeling $f$ and having vertices $u_{1}$ and $u_{n_{1}}$ with the labels 1 and $n_{1}$ respectively. Then the graph $G$ obtained by identifying an end vertex of a path $P_{n_{2}}$ with either $u_{1}$ or $u_{n_{1}}$ of $G_{1}$ is a prime graph.

Proof: Let the vertices of a prime graph $G_{1}$ be $u_{1}, u_{2}, \cdots, u_{n_{1}}$ and the prime labeling $f$ of $G_{1}$ be such that $f\left(u_{1}\right)=1$ and $f\left(u_{n_{1}}\right)=n_{1}$. Let $v_{1}, v_{2}, \cdots, v_{n_{2}}$ be the consecutive vertices of $P_{n_{2}}$. We have the following two cases: 
1. The graph $G$ is obtained by identifying an end vertex $v_{1}$ of $P_{n_{2}}$ with the vertex $u_{n_{1}}$ of $G_{1}$. (The proof is similar if the other end vertex $v_{n_{2}}$ of $P_{n_{2}}$ is identified with the vertex $u_{n_{1}}$ of $G_{1}$.) Define a labeling function $g$ on $G$ as follows:

$g\left(u_{i}\right)=f\left(u_{i}\right), \forall i=1,2,3, \cdots, n_{1} ;$ and $g\left(v_{j}\right)=n_{1}+j-1, \forall j=2,3,4, \cdots, n_{2}$.

Obviously $g$ is an injection. Also $g$ is an extension of the prime labeling function $f$ on $G$, it is enough to prove the following cases:

(a) $\operatorname{gcd}\left(g\left(u_{n_{1}}\right), g\left(v_{2}\right)\right)=1$. To prove this we have $\operatorname{gcd}\left(g\left(u_{n_{1}}\right), g\left(v_{2}\right)\right)=$ $\operatorname{gcd}\left(f\left(u_{n_{1}}\right), g\left(v_{2}\right)\right)=\operatorname{gcd}\left(n_{1}, n_{1}+1\right)=1$ as $n_{1}$ and $n_{1}+1$ are consecutive integers.

(b) For each $j \in\left\{2,3, \cdots, n_{2}-1\right\}, \operatorname{gcd}\left(g\left(v_{j}\right), g\left(v_{j+1}\right)\right)=1$. To prove this we have $\operatorname{gcd}\left(g\left(v_{j}\right), g\left(v_{j+1}\right)\right)=\operatorname{gcd}\left(n_{1}+\right.$ $\left.j-1, n_{1}+j\right)=1$ as $n_{1}+j-1$ and $n_{1}+j$ are consecutive integers.

2. The graph $G$ is obtained by identifying the other end vertex $v_{1}$ of $P_{n_{2}}$ with the vertex $u_{1}$ of $G_{1}$. (The proof is similar if the other end vertex $v_{n_{2}}$ of $P_{n_{2}}$ is identified with the vertex $u_{1}$ of $G_{1}$.)

Define a labeling function $g$ on $G$ as follows:

$g\left(u_{i}\right)=f\left(u_{i}\right), \forall i=1,2,3, \cdots, n_{1} ;$ and $g\left(v_{j}\right)=n_{1}+j-1, \forall j=2,3,4, \cdots, n_{2}$.

Obviously $g$ is an injection. Also $g$ is an extension of the prime labeling function $f$ on $G$, it is enough to prove the following cases:

(a) $\operatorname{gcd}\left(g\left(u_{1}\right), g\left(v_{2}\right)\right)=1$. To prove this we have $\operatorname{gcd}\left(g\left(u_{1}\right), g\left(v_{2}\right)\right)=\operatorname{gcd}\left(f\left(u_{1}\right), g\left(v_{2}\right)\right)=\operatorname{gcd}\left(1, n_{1}+1\right)=1$.

(b) For each $j \in\left\{2,3, \cdots, n_{2}-1\right\}$ we need to show that $\operatorname{gcd}\left(g\left(v_{j}\right), g\left(v_{j+1}\right)\right)=1$. To prove this we have $\operatorname{gcd}\left(g\left(v_{j}\right), g\left(v_{j+1}\right)\right)=\operatorname{gcd}\left(n_{1}+j-1, n_{1}+j\right)=1$ as $n_{1}+j-1$ and $n_{1}+j$ are consecutive integers.

Thus in each of the possibilities the graph $G$ under consideration admits a prime labeling. Which implies that $G$ is a prime graph.

Theorem 2.10 A graph $G$ obtained by identifying all the apex vertices of $m$ fans $f_{n_{1}}, f_{n_{2}}, \cdots, f_{n_{m}}$ ( is called a multiple shell) is a prime graph.

Proof: A fan graph $f_{n}=P_{n-1}+K_{1}$ has $n$ vertices and $2 n-3$ edges. Let the graph $G$ is obtained by fusing all the apex vertices of $f_{n_{1}}, f_{n_{2}}, \cdots, f_{n_{m}}$. Let the common apex vertex of each of the fans $f_{n_{i}}$ after fusing all the apex vertices of all the fans $f_{n_{1}}, f_{n_{2}}, \cdots, f_{n_{m}}$ be $v_{0}$. For each $i \in\{1,2,3, \cdots, m\}$, denote the remaining vertices of the fan $f_{n_{i}}$ as $v_{i, 1}, v_{i, 2}, \cdots, v_{i, n_{i-1}}$ consecutively. Clearly $|V|=n_{1}+n_{2}+\cdots+n_{m}-m+1$. Without loss of generality assume that $n_{1} \leq n_{2} \leq \cdots \leq n_{m}$. Define $f: V \rightarrow\{1,2, \cdots,|V|\}$ as follows:

$f\left(v_{0}\right)=1$ and $f\left(v_{i, j}\right)=\sum_{k=1}^{i-1} n_{k}-(i-2)+j, \forall i=1,2, \cdots, m$ and $\forall j=1,2, \cdots, n_{i}-1$. Here we define $\sum_{x}^{y} a=0$ if $x$ and $y$ are any positive integers with $y<x$. First we will show that $f$ is an injection. It is easy to check that $f(v)=1$ if and only if $v=v_{0}$. For $i \in\{1,2, \cdots, m-1\}, j \in\left\{1,2, \cdots, n_{i+1}-1\right\}$ and $j^{\prime} \in\left\{1,2, \cdots, n_{i}-1\right\}$, we get

$$
\begin{aligned}
f\left(v_{i+1, j}\right)-f\left(v_{i, j^{\prime}}\right) & =\left(\sum_{k=1}^{i} n_{k}-(i+1-2)+j\right)-\left(\sum_{k=1}^{i-1} n_{k}-(i-2)+j^{\prime}\right) \\
& =\left(n_{i}-1-j^{\prime}\right)+j \\
& \geq j \text { as } 1 \leq j^{\prime} \leq n_{i}-1 .
\end{aligned}
$$

Thus $f\left(v_{i+1, j}\right)-f\left(v_{i, j^{\prime}}\right)>0$. Thus if $i<i^{\prime}$ with $i, i^{\prime} \in\{1,2, \cdots, m\}$ then $f\left(v_{i, j}\right)<f\left(v_{i^{\prime}, j^{\prime}}\right), \forall j \in\left\{1,2, \cdots, n_{i}-1\right\}$ and $j^{\prime} \in\left\{1,2, \cdots, n_{i}^{\prime}-1\right\}$. If $i \neq i^{\prime}$ then without loss of generality assume that $i<i^{\prime} . i<i^{\prime} \Rightarrow f\left(v_{i, j}\right)<f\left(v_{i, j^{\prime}}\right)$. Similarly we have $i^{\prime}<i \Rightarrow f\left(v_{i^{\prime}, j^{\prime}}\right)<f\left(v_{i, j}\right)$. That is $f\left(v_{i^{\prime}, j^{\prime}}\right)=f\left(v_{i, j}\right) \Rightarrow i^{\prime}=i$. If $i=i^{\prime}$ then $f\left(v_{i, j}\right)=f\left(v_{i^{\prime}, j^{\prime}}\right) \Rightarrow f\left(v_{i, j}\right)=f\left(v_{i, j^{\prime}}\right)$. $\Rightarrow \sum_{k=1}^{i-1} n_{k}-(i-2)+j=\sum_{k=1}^{i-1} n_{k}-(i-2)+j^{\prime} \Rightarrow j=j^{\prime}$. Which shows that $f$ is an injection. It is enough to show that $f$ is a prime labeling. Let $e=u v$ be an edge of $G$. Then clearly it must be an edge of exactly one of the fans $f_{n_{i}}$ for some $i=1,2, \cdots, m$. If one of the end vertices of $e$ is $v_{0}$ say $u=v_{0}$ then $\operatorname{gcd}(f(u), f(v))=\operatorname{gcd}\left(f\left(v_{0}\right), f(v)\right)=\operatorname{gcd}(1, f(v))=1$. If none of the end vertices of $e$ is $v_{0}$ then clearly $\{u, v\}=\left\{v_{i, j}, v_{i, j+1}\right\}$ for some $i \in\{1,2, \cdots, m\}$ and $j \in\left\{1,2, \cdots, n_{i}-1\right\}$.

$$
\text { That is } \begin{aligned}
\operatorname{gcd}(f(u), f(v)) & =\operatorname{gcd}\left(f\left(v_{i, j}\right), f\left(v_{i, j+1}\right)\right) \\
& =\operatorname{gcd}\left(\sum_{k=1}^{i-1} n_{k}-(i-2)+j, \sum_{k=1}^{i-1} n_{k}-(i-2)+j+1\right) \\
& =1
\end{aligned}
$$

as consecutive integers are relatively prime. Thus $f$ admits a prime labeling for $G$. i.e. $G$ is a prime graph. 
Illustration 2.11 The graph $G$ obtained by identifying all the apex vertices of three fans $f_{3}, f_{4}, f_{5}$ has prime labeling is shown in Figure 5.

Theorem 2.12 A graph $G$ obtained by identifying all the apex vertices of $m$ wheels $W_{n_{1}}, W_{n_{2}}, \cdots, W_{n_{m}}$ is a prime graph if each $n_{i} \geq 4$ is an even integer for each $i \in\{1,2, \cdots, m\}$ and $n_{i}-1$ is relatively prime with $2+\sum_{k=1}^{i-1} n_{k}$ for each $i \in\{2,3, \cdots, m\}$.

Proof: Let the common apex vertex of $G$ be $u_{0}$ and the consecutive rim vertices of each of the wheels $W_{n_{i}}$ be $u_{i, 1}, u_{i, 2}, \cdots$, $u_{i, n_{i}}$ for each $i \in\{1,2, \cdots, m\}$. Here we define $\sum_{x}^{y} a=0$ if $x$ and $y$ are any positive integers with $y<x$.

Define $f:\left\{u_{0}\right\} \bigcup\left(\bigcup_{i=1}^{m}\left\{u_{i, 1}, u_{i, 2}, u_{i, 3}, \cdots, u_{i, n_{i}}\right\}\right) \rightarrow\left\{1,2, \cdots, 1+\sum_{i=1}^{m} n_{i}\right\}$ as

$$
f(x)= \begin{cases}1 & \text {,if } x=u_{0} \\ 1+j+\sum_{k=1}^{i-1} n_{k} & , \text { if } x=u_{i, j} \text { for some } j \in\left\{1,2, \cdots, n_{i}\right\}, i \in\{1,2, \cdots, m\}\end{cases}
$$

To prove $f$ is injective it is enough to prove that $f$ is surjective as the cardinality of the domain and codomain are same. For each $y \in\left\{1,2, \cdots, 1+\sum_{i=1}^{m} n_{i}\right\}$ either $y=1=f\left(u_{0}\right)$ or there exists $i \in\{1,2, \cdots, m\}$ such that $1+\sum_{k=1}^{i-1} n_{k}<y \leq 1+\sum_{k=1}^{i} n_{k}$.

$$
\begin{aligned}
\text { For letter case } j & =y-\left(1+\sum_{k=1}^{i-1} n_{k}\right), \text { then } 1 \leq j \leq n_{i} \\
\text { and } f\left(u_{i, j}\right) & =1+j+\sum_{k=1}^{i-1} n_{k} \\
& =1+y-\left(1+\sum_{k=1}^{i-1} n_{k}\right)+\sum_{k=1}^{i-1} n_{k} \\
& =y .
\end{aligned}
$$

It shows that $f$ is surjective. Let $e=x y$ be an edge of $G$ then it must be an edge of one of the wheels $W_{n_{i}}$ for some $i \in\{1,2, \cdots, m\}$. we have the following two possibilities:

1. If one of the end vertices of $e$ is the apex vertex $u_{0}$ with $x=u_{0}$ then $\operatorname{gcd}(f(x), f(y))=\operatorname{gcd}\left(f\left(u_{0}\right), f(y)\right)=\operatorname{gcd}(1, f(y))=1$.

2. If none of the end vertices of $e$ is the apex vertex $u_{0}$ and

- if $\{x, y\}=\left\{u_{i, j-1}, u_{i, j}\right\}$ for some $j \in\left\{1,2, \cdots, n_{i}\right\}$ then

$$
\begin{aligned}
\operatorname{gcd}(f(x), f(y)) & =\operatorname{gcd}\left(f\left(u_{i, j-1}\right), f\left(u_{i, j}\right)\right) \\
& =\operatorname{gcd}\left(j+\sum_{k=1}^{i-1} n_{k}, 1+j+\sum_{k=1}^{i-1} n_{k}\right) \\
& =1
\end{aligned}
$$

as $j+\sum_{k=1}^{i-1} n_{k}$ and $1+j+\sum_{k=1}^{i-1} n_{k}$ are consecutive integers so they necessarily be relatively prime.

- if $\{x, y\}=\left\{u_{i, 1}, u_{i, n_{i}}\right\}$ then

$$
\begin{aligned}
\operatorname{gcd}(f(x), f(y)) & =\operatorname{gcd}\left(f\left(u_{i, 1}\right), f\left(u_{i, n_{i}}\right)\right) \\
& =\operatorname{gcd}\left(1+1+\sum_{k=1}^{i-1} n_{k}, 1+n_{i}+\sum_{k=1}^{i-1} n_{k}\right) \\
& =\operatorname{gcd}\left(2+\sum_{k=1}^{i-1} n_{k}, n_{i}-1\right) \\
& =1
\end{aligned}
$$


as it is mentioned that for each $i \in\{2,3, \cdots, m\}, n_{i}-1$ is relatively prime with $2+\sum_{k=1}^{i-1} n_{k}$.

Which shows that $f$ admits a prime labeling i.e. $G$ is a prime graph.

Illustration 2.13 The graph $G$ obtained by identifying the apex vertices of two wheels $W_{6}$ and $W_{8}$ has prime labeling is shown in Figure 6.

Theorem 2.14 A $t$-ply graph $P_{t}(u, v)$ is a prime graph if the order of $P_{t}(u, v)$ is a prime number.

Proof: Suppose a $t$-ply $P(u, v)$ is obtained from $t$ distinct paths $P_{i}$, for each $i=1,2, \cdots, t$, each of length $n_{i}$, such that the vertices of $P_{i}$ are $v_{i, 0}, v_{i, 1}, v_{i, 2}, \cdots, v_{i, n_{i}}$ consecutively. Identifying all the vertices $v_{1,0}, v_{2,0}, v_{3,0}, \cdots, v_{t, 0}$ into a single vertex $u$ and identifying all the vertices $v_{1, n_{1}}, v_{2, n_{2}}, v_{3, n_{3}}, \cdots, v_{t, n_{t}}$ into a single vertex $v$. The number of vertices of $P_{t}(u, v)$ is a prime number $p$ with

$$
\begin{aligned}
p & =\left|V\left(P_{t}(u, v)\right)\right| \\
& =\sum_{i=1}^{t}\left(\left|V\left(P_{i}\right)\right|-2\right)+2 \\
& =\sum_{i=1}^{t}\left(n_{i}+1\right)-2 t+2 \\
& =\sum_{i=1}^{t} n_{i}-t+2 .
\end{aligned}
$$

Define $f: V\left(P_{t}(u, v)\right) \rightarrow\{1,2,3, \cdots, p\}$ as follows: $f(u)=1$ and $f\left(v_{i, j}\right)=j+\sum_{k=1}^{i-1}\left(n_{k}-1\right), \forall i=1,2, \cdots, t$ and $\forall j=1,2, \cdots, n_{i}-1$ and $f(v)=p$ where $p$ is a prime number. Here we define $\sum_{x}^{y} a=0$ if $x$ and $y$ are any positive integers with $y<x$. First we will show that $f$ is an injection. It is easy to check that $f(w)=1$ if and only if $w=u$ as well as $f(w)=p$ if and only if $w=v$. Suppose $f\left(v_{i, j}\right)=f\left(v_{i^{\prime}, j^{\prime}}\right)$ for some positive integers $i, j, i^{\prime}, j^{\prime}$ with $i \in\{1,2, \cdots, t\}$, $i^{\prime} \in\{1,2, \cdots, t\}$ and $j \in\left\{1,2, \cdots, n_{i}-1\right\}$ and $j^{\prime} \in\left\{1,2, \cdots, n_{i}^{\prime}-1\right\}$. If $i \neq i^{\prime}$ then without loss of generality assume that $i<i^{\prime}$, so $i \leq i^{\prime}-1$.

$$
\text { Then } \begin{aligned}
f\left(v_{i, j}\right) & =j+\sum_{k=1}^{i-1}\left(n_{k}-1\right) \\
& \leq n_{i}-1+\sum_{k=1}^{i-1}\left(n_{k}-1\right) \\
& =\sum_{k=1}^{i}\left(n_{k}-1\right) \\
& \leq \sum_{k=1}^{i^{\prime}-1}\left(n_{k}-1\right) \\
& <j^{\prime}+\sum_{k=1}^{i^{\prime}-1}\left(n_{k}-1\right) \\
& =f\left(v_{i^{\prime}, j^{\prime}}\right) .
\end{aligned}
$$

Thus we have $i<i^{\prime} \Rightarrow f\left(v_{i, j}\right)<f\left(v_{i, j^{\prime}}\right)$ and similarly $i^{\prime}<i \Rightarrow f\left(v_{i^{\prime}, j^{\prime}}\right)<f\left(v_{i, j}\right)$.

$$
\text { That is } \begin{aligned}
f\left(v_{i, j}\right)=f\left(v_{i^{\prime}, j^{\prime}}\right) & \Rightarrow i=i^{\prime} \\
& \Rightarrow f\left(v_{i, j}\right)=f\left(v_{i, j^{\prime}}\right) \\
& \Rightarrow j+\sum_{k=1}^{i-1}\left(n_{k}-1\right)=j^{\prime}+\sum_{k=1}^{i-1}\left(n_{k}-1\right) \\
& \Rightarrow j=j^{\prime} .
\end{aligned}
$$

Thus $f\left(v_{i, j}\right)=f\left(v_{i^{\prime}, j^{\prime}}\right) \Rightarrow i=i^{\prime}, j=j^{\prime}$, which shows that $f$ is injective. 
It is enough to show that $\operatorname{gcd}(f(x), f(y))=1$, for every pair of adjacent vertices $x$ and $y$. Let $e=x y$ be an edge of $G$. Then clearly it must be an edge of exactly one of the paths $P_{n_{i}}$ for some $i=1,2, \cdots, t$.

1. If one of the end vertices of $e$ is $u$ say $x=u$ then $\operatorname{gcd}(f(x), f(y))=\operatorname{gcd}(f(u), f(y))=\operatorname{gcd}(1, f(y))=1$.

2. If one of the end vertices of $e$ is $v$ say $x=v$ then $\operatorname{gcd}(f(x), f(y))=\operatorname{gcd}(f(v), f(y))=\operatorname{gcd}(p, f(y))=1$.

3. If none of the end vertices of $e$ is $u$ and $v$ then clearly $\{u, v\}=\left\{v_{i, j}, v_{i, j+1}\right\}$ for some $j \in\left\{1,2, \cdots, n_{i}-2\right\}$.

$$
\text { i.e. } \quad \begin{aligned}
\operatorname{gcd}(f(x), f(y)) & =\operatorname{gcd}\left(f\left(v_{i, j}\right), f\left(v_{i, j+1}\right)\right) \\
& =\operatorname{gcd}\left(j+\sum_{k=1}^{i-1}\left(n_{k}-1\right), j+1+\sum_{k=1}^{i-1}\left(n_{k}-1\right)\right) \\
& =1
\end{aligned}
$$

as any two consecutive integers are relatively prime.

Thus $f$ admits a prime labeling for $G$. That is $G$ is a prime graph.

Illustration 2.15 The 5-ply graph obtained by taking five paths of lengths 6, 6, 4, 6 and 10 respectively has prime labeling is shown in Figure 7.

\section{3. $k$-Prime Labeling- a New Concept}

Definition 3.1 A $k$-prime labeling of a graph $G$ is an injective function $f: V \rightarrow\{k, k+1, k+2, k+3, \cdots, k+|V|-1\}$ for some positive integer $k$ that induces a function $f^{+}: E(G) \rightarrow N$ of the edges of $G$ defined by $f^{+}(u v)=\operatorname{gcd}(f(u), f(v))$, $\forall e=u v \in E(G)$ such that $\operatorname{gcd}(f(u), f(v))=1, \forall e=u v \in E(G)$. The graph which admits a $k$-prime labeling is called a $k$-prime graph.

One note that every prime graph is a $k$-prime graph for $k=1$.

Lemma 3.2 For each positive integer $m$ the path graph $P_{m}$ is a $k$-prime graph for each positive integer $k$.

Proof: Denote the vertices of $P_{m}$ as $v_{1}, v_{2}, \cdots, v_{m}$ in the order. For each positive integer $k$, define $f: V \rightarrow\{k, k+1, k+$ $2, k+3, \cdots, k+|V|-1\}$ as $f\left(v_{i}\right)=k+i-1$ for each $v_{i} \in V$. For $\forall e=u v \in E(G), f$ induces a function $f^{+}: E(G) \rightarrow N$ defined by $f^{+}(u v)=\operatorname{gcd}(f(u), f(v))$. $\forall e=v_{i} v_{i+1} \in E(G)$ it is easy to deduce that $\operatorname{gcd}\left(f\left(v_{i}\right), f\left(v_{i+1}\right)\right)=\operatorname{gcd}(k+i-1, k+i)=$ 1. Thus the path graph $P_{m}$ is a $k$-prime graph.

Theorem 3.3 The graph $G$ obtained by disjoint union of a prime graph $G_{1}$ of order $n_{1}$ and a $\left(n_{1}+1\right)-$ prime graph $G_{2}$ is a prime graph.

Proof: Let $f_{1}:\left\{u_{1}, u_{2}, \cdots, u_{n_{1}}\right\} \longrightarrow\left\{1,2, \cdots, n_{1}\right\}$ be a prime labeling of a prime graph $G_{1}$. Let $f_{2}:\left\{v_{1}, v_{2}, \cdots, v_{n_{2}}\right\} \longrightarrow$ $\left\{n_{1}+1, n_{1}+2, n_{1}+3, \cdots, n_{1}+n_{2}\right\}$ be a $\left(n_{1}+1\right)$-prime labeling of a $\left(n_{1}+1\right)$-prime graph $G_{2}$. Let the graph $G$ be obtained by disjoint union of $G_{1}$ and $G_{2}$.

Define $f:\left\{u_{1}, u_{2}, \cdots, u_{n_{1}}\right\} \cup\left\{v_{1}, v_{2}, \cdots, v_{n_{2}}\right\} \rightarrow\left\{1,2, \cdots, n_{1}+n_{2}\right\}$ as

$$
f(x)= \begin{cases}f_{1}\left(u_{i}\right) & , \text { if } x=u_{i} \text { for some } i \in\left\{1,2, \cdots, n_{1}\right\} \\ f_{2}\left(v_{j}\right) & , \text { if } x=v_{j} \text { for some } j \in\left\{1,2, \cdots, n_{2}\right\}\end{cases}
$$

Obviously $f$ is an injection. Let $e=x y$ be an arbitrary edge of $G$. Then either $e \in E\left(G_{1}\right)$ or $e \in E\left(G_{2}\right)$.

1. If $e \in E\left(G_{1}\right)$ then $\operatorname{gcd}(f(x), f(y))=\operatorname{gcd}\left(f_{1}(x), f_{1}(y)\right)=1$, as $f_{1}$ is a prime labeling of $G_{1}$.

2. If $e \in E\left(G_{2}\right)$ then $\operatorname{gcd}(f(x), f(y))=\operatorname{gcd}\left(f_{2}(x), f_{2}(y)\right)=1$, as $f_{2}$ is a prime labeling of $G_{2}$.

Thus $f$ admits a prime labeling of $G$ and consequently $G$ is a prime graph.

Theorem 3.4 Let $G_{1}$ be a prime graph of order $n_{1}$ with a prime labeling $f_{1}$ and having vertices $u_{1}$ and $u_{n_{1}}$ with $f_{1}\left(u_{1}\right)=1$ and $f_{1}\left(u_{n_{1}}\right)=n_{1}$. Let $G_{2}$ be a $n_{1}$-prime graph of order $n_{2}$ with a $n_{1}$-prime labeling $f_{2}$ having a vertex $v_{1}$ with $f_{2}\left(v_{1}\right)=n_{1}$. Then the graph $G$ obtained by identifying the vertex $v_{1}$ of $G_{2}$ with either to $u_{1}$ or to $u_{n_{1}}$ of $G_{1}$ is a prime graph.

Proof: Let $f_{1}:\left\{u_{1}, u_{2}, \cdots, u_{n_{1}}\right\} \longrightarrow\left\{1,2, \cdots, n_{1}\right\}$ be a prime labeling of a prime graph $G_{1}$. Let $f_{2}:\left\{v_{1}, v_{2}, \cdots, v_{n_{2}}\right\} \longrightarrow$ $\left\{n_{1}, n_{1}+1, n_{1}+2, \cdots, n_{1}+n_{2}-1\right\}$ be a $n_{1}$-prime labeling of a $n_{1}$-prime graph $G_{2}$.

1. Consider the graph $G$ obtained by identifying the vertex $v_{1}$ of $G_{2}$ to $u_{1}$ of $G_{1}$. 
Define $f:\left\{u_{1}, u_{2}, \cdots, u_{n_{1}}, v_{2}, v_{3}, \cdots, v_{n_{2}}\right\} \longrightarrow\left\{1,2, \cdots, n_{1}+n_{2}\right\}$ as

$$
f(x)= \begin{cases}f_{1}\left(u_{i}\right) & , \text { if } x=u_{i} \text { for some } i \in\left\{1,2, \cdots, n_{1}\right\} \\ f_{2}\left(v_{j}\right) & , \text { if } x=v_{j} \text { for some } j \in\left\{2,3, \cdots, n_{2}\right\}\end{cases}
$$

We claim that $f$ is an injection because

- $f_{1}$ is an injection from $\left\{u_{1}, u_{2}, \cdots, u_{n_{1}}\right\}$ with the range $\left\{1,2, \cdots, n_{1}\right\}$.

- $f_{2}$ is an injection from $\left\{v_{1}, v_{2}, \cdots, v_{n_{2}}\right\}$ with the range $\left\{n_{1}, n_{1}+1, \cdots \cdots, n_{1}+n_{2}-1\right\}$ and $f_{2}\left(v_{1}\right)=n_{1}$ then the restriction of $f_{2}$ on $\left\{v_{2}, v_{3}, \cdots, v_{n_{2}}\right\}$ is also an injection with the range $\left\{n_{1}+1, n_{1}+2, \cdots, n_{1}+n_{2}-1\right\}$.

Let $e=x y$ be an arbitrary edge of $G$. Then either $e \in E\left(G_{1}\right)$ or $e \in E\left(G_{2}\right)$.

(a) If $e \in E\left(G_{1}\right)$ then $\operatorname{gcd}(f(x), f(y))=\operatorname{gcd}\left(f_{1}(x), f_{1}(y)\right)=1$, as $f_{1}$ is a prime labeling of $G_{1}$.

(b) If $e \in E\left(G_{2}\right)$ then $\operatorname{gcd}(f(x), f(y))=\operatorname{gcd}\left(f_{2}(x), f_{2}(y)\right)=1$ as $f_{2}$ is a prime labeling of $G_{2}$.

2. Consider the graph $G$ obtained by identifying the vertex $v_{1}$ of $G_{2}$ to $u_{n_{1}}$ of $G_{1}$.

Define $f:\left\{u_{1}, u_{2}, \cdots, u_{n_{1}}, v_{2}, v_{3}, \cdots, v_{n_{2}}\right\} \rightarrow\left\{1,2, \cdots, n_{1}+n_{2}\right\}$ as

$$
f(x)= \begin{cases}f_{1}\left(u_{i}\right) & , \text { if } x=u_{i} \text { for some } i \in\left\{1,2, \cdots, n_{1}\right\} \\ f_{2}\left(v_{j}\right) & , \text { if } x=v_{j} \text { for some } j \in\left\{2,3, \cdots, n_{2}\right\}\end{cases}
$$

We claim that $f$ is an injection because

- $f_{1}$ is an injection from $\left\{u_{1}, u_{2}, \cdots, u_{n_{1}}\right\}$ with the range $\left\{1,2, \cdots, n_{1}\right\}$.

- $f_{2}$ is an injection from $\left\{v_{1}, v_{2}, \cdots, v_{n_{2}}\right\}$ with the range $\left\{n_{1}, n_{1}+1, \cdots \cdots, n_{1}+n_{2}-1\right\}$ and $f_{2}\left(v_{1}\right)=n_{1}$ then the restriction of $f_{2}$ on $\left\{v_{2}, v_{3}, \cdots, v_{n_{2}}\right\}$ is also an injection with the range $\left\{n_{1}+1, n_{1}+2, \cdots, n_{1}+n_{2}-1\right\}$.

Let $e=x y$ be an arbitrary edge of $G$. Then either $e \in E\left(G_{1}\right)$ or $e \in E\left(G_{2}\right)$.

(a) If $e \in E\left(G_{1}\right)$ then $\operatorname{gcd}(f(x), f(y))=\operatorname{gcd}\left(f_{1}(x), f_{1}(y)\right)=1$ as $f_{1}$ is a prime labeling of $G_{1}$.

(b) If $e \in E\left(G_{2}\right)$ then $\operatorname{gcd}(f(x), f(y))=\operatorname{gcd}\left(f_{2}(x), f_{2}(y)\right)=1$ as $f_{2}$ is a prime labeling of $G_{2}$.

Thus in each of the possibilities $f$ admits a prime labeling of $G$ consequently $G$ is a prime graph.

Corollary 3.5 A tadpole (graph obtained by identifying a vertex of a cycle to an end vertex of a path) is a prime graph.

Proof: As we know that every cycle is a prime graph. According to Lemma 3.2 every path is $k$-prime graph for every positive integer $k$. Then using Theorem 3.4 a tadpole is a prime graph.

\section{Conclusion}

Here we investigate eight results corresponding to prime labeling. We introduce a new concept of $k$-prime labeling and derive four results. Analogous work can be carried out for other families and in the context of different types of graph labeling techniques.

\section{References}

Burton, D. M. (1990). Elementary Number Theory. 2nd ed.

Deretsky, T.D., Lee, S.M., \& Mitchem, J. (1991). On vertex prime labellings of graphs, Graph Theory, Combinatorics and Applications, Vol. 1, (Ed. J. Alvi, G. Chartrand, O. Oellerman, A. Schwenk), Proceedings of the 6th International Conference Theory and Applications of Graphs (pp. 359-369)

Fu, H. L. \& Huang, K. C. (1994). On Prime Labelings. Discrete Mathematics, North Holland, 127, 181-186

Gallian, J. A. (2009). A Dynamic Survey of Graph Labeling The Electronic Journal of Combinatorics, 16 , \#DS6.

Gross, J. \& Yellen, J. (2004). Handbook of Graph Theory, CRC Press.

Lee, S. M., Wui, I. \& Yeh, J. (1988). On the amalgamation of prime graphs. Bull. Malaysian Math. Soc.( Second Series), 11, pp 59-67.

Tout, A., Dabboucy, A. N. \& and Howalla, K. (1982). Prime Labeling of Graphs. Nat. Acad. Sci. Letters, 11, pp. $365-368$. 


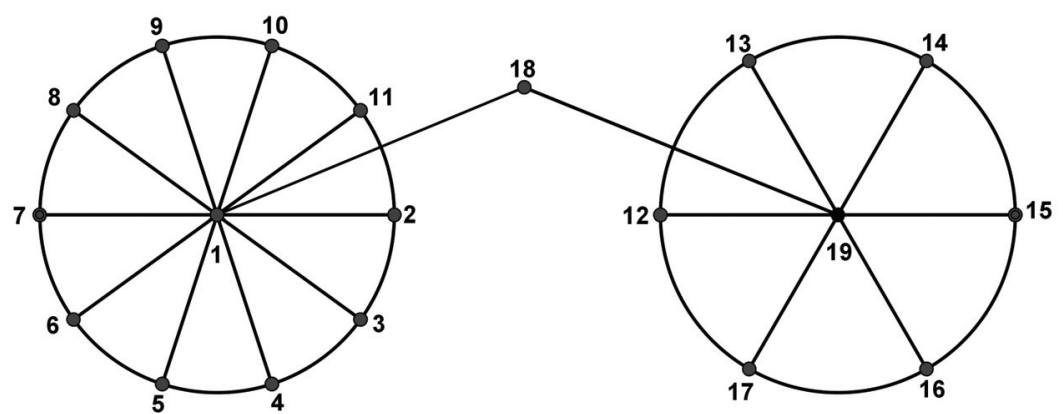

Figure 1. Prime labeling of $<W_{10}: W_{6}>$ is shown.
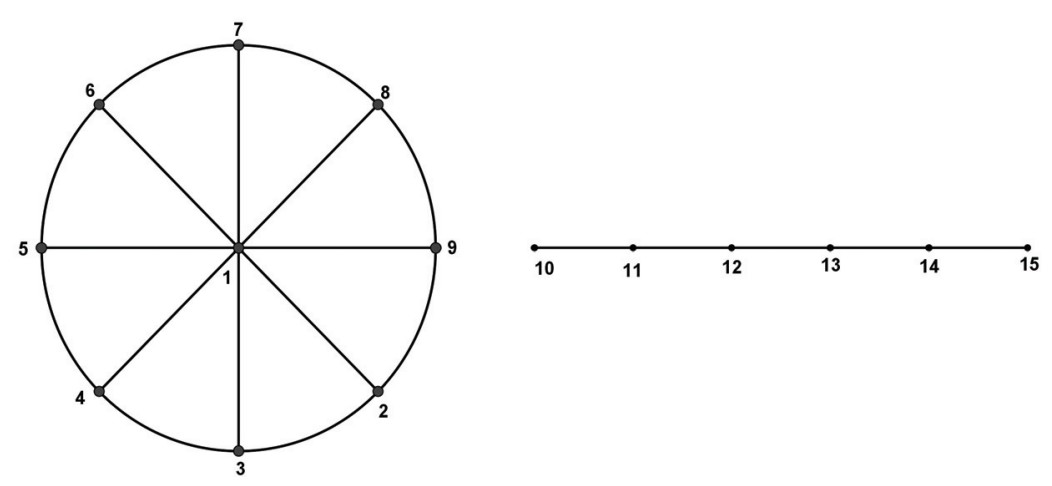

Figure 2. The disjoint union of $W_{8}$ and $P_{6}$ and its prime labeling

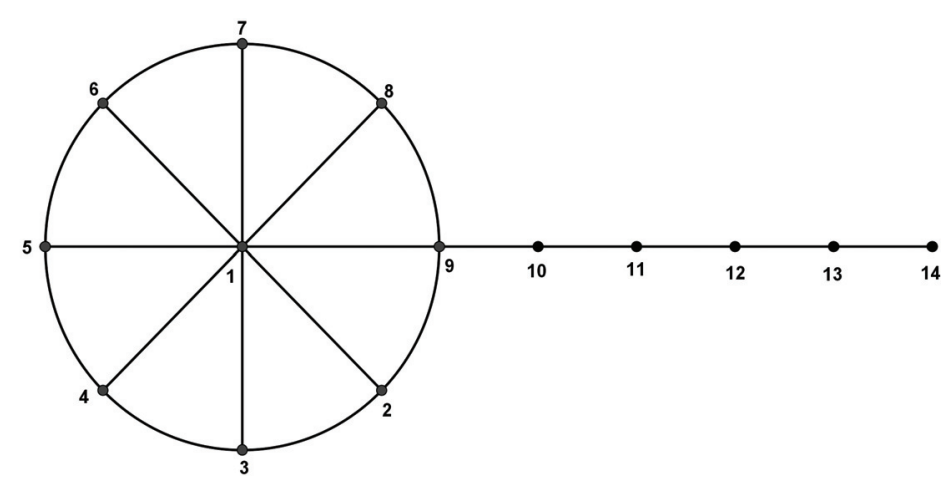

Figure 3. Graph obtained by identifying a rim vertex of $W_{8}$ with an end vertex of $P_{6}$ and its prime labeling.

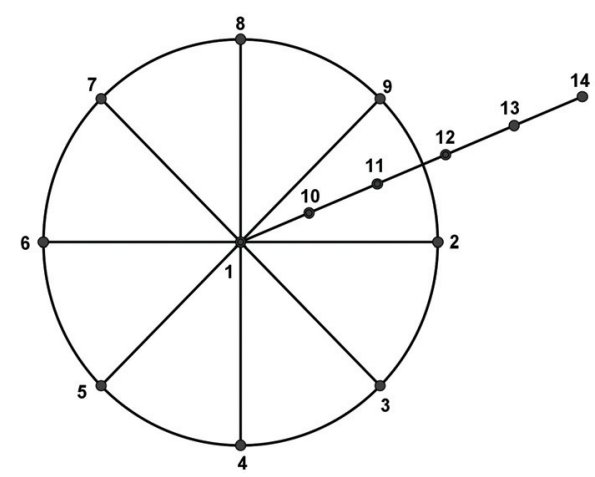

Figure 4. The graph obtained by identifying the apex vertex of $W_{8}$ with end vertex of $P_{6}$ and its prime labeling 


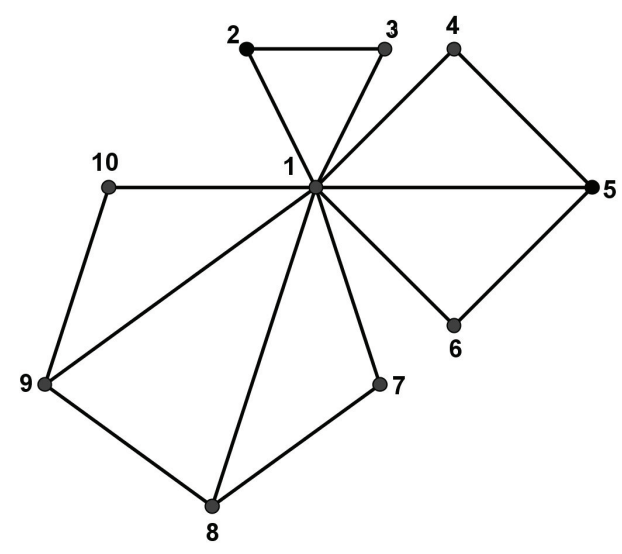

Figure 5. Graph obtained by identifying the apex vertices of $f_{3}, f_{4}, f_{5}$ and its prime labeling.

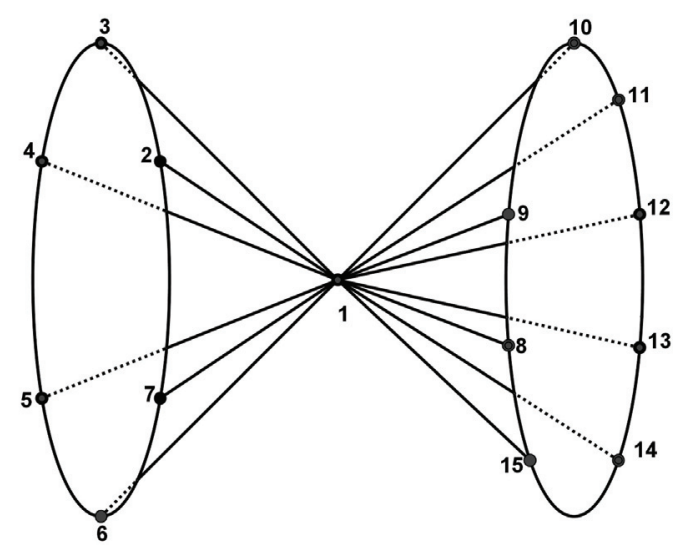

Figure 6. The graph obtained by identifying the apex vertices of $W_{6}$ and $W_{8}$ and its prime labeling.

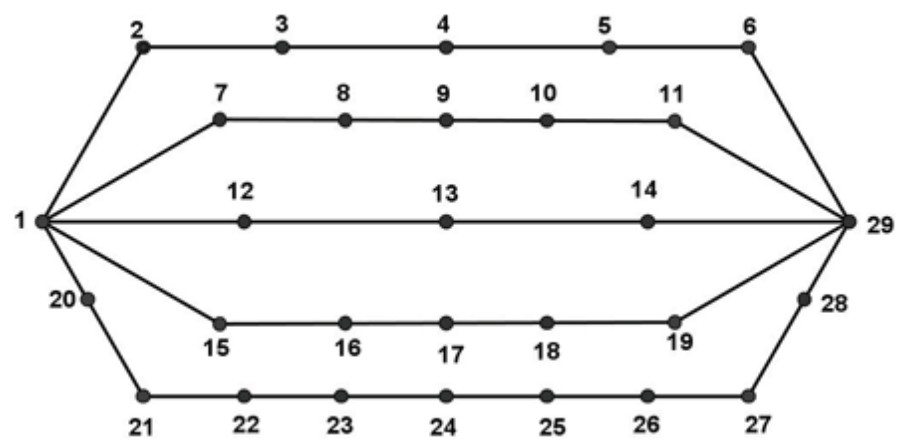

Figure 7. A 5-ply and its prime labeling 